\title{
The effect of subadditive pretraining on blocking: Limits on generalization
}

\author{
DANIEL S. WHEELER \\ State University of New York, Binghamton, New York \\ TOM BECKERS \\ University of Leuven, Leuven, Belgium \\ AND \\ RALPH R. MILler \\ State University of New York, Binghamton, New York
}

\begin{abstract}
Recent evidence indicates that prior learning about a set of cues may determine how new cues are processed. If subjects are taught that two reliable predictors of an outcome do not summate when the cues are presented together (i.e., subadditive pretraining), the subjects will tend to show a less profound blocking effect when trained with different cues. Three experiments investigated the conditions necessary for subadditive pretraining to generalize to new cues. Experiment 1 demonstrated that subadditive pretraining is less effective in reducing blocking when it is experienced in a context other than that in which the blocking training is experienced. In Experiment 2, the effectiveness of subadditive pretraining waned with time. Experiment 3 showed that subadditive pretraining is more effective when the temporal characteristics of pretraining cues are similar to those of the cues used in blocking training. These results provide information concerning the conditions under which learning will generalize from one set of cues to another.
\end{abstract}

Associative learning theories are widely applied in the fields of Pavlovian conditioning and human contingency learning. In general, they posit that the contingency between a given cue and an outcome is encoded as the formation and strengthening of an association between mental representations of the cue and the outcome. Moreover, associative theories often posit that processing occurs in a bottom-up fashion. Previously acquired knowledge will only affect the processing of a stimulus if that knowledge pertains to an associative structure that involves the stimulus or one of its direct associates. However, there have been a number of recent observations that challenge basic associative theories by suggesting the potential influence of higher order top-down processes in learning. In particular, recent studies have suggested that the Kamin (1968) blocking effect can be affected by previous learning about cues that are not involved in blocking training.

Blocking manifests as a reduction in responding to a target cue when it is paired with an outcome in the presence of a previously established, reliable predictor of the outcome (i.e., a blocking cue). Procedurally, training that typically results in blocking can be represented as A+ trials followed by $\mathrm{AX}+$ trials, in which $\mathrm{A}$ is the blocking cue, $\mathrm{X}$ is the target cue, and "+" denotes the presence of the outcome. This phenomenon has been observed in
Pavlovian conditioning situations in which conditioned stimuli (CSs) serve as cues and an unconditioned stimulus (US) serves as the outcome (e.g., Hinchy, Lovibond, \& Ter-Horst, 1995; Kamin, 1968). Blocking has also been demonstrated in human contingency learning preparations in which participants are asked to judge the contingency between cues and outcomes that are more representational in nature (i.e., words and/or pictures are used to represent cues and outcomes; e.g., Shanks \& Dickinson, 1987). Associative accounts of blocking use different mechanisms, but most theories suggest that the presence of a blocking cue impairs the acquisition of a strong target cue-outcome association. For example, the Rescorla-Wagner (1972) model states that the acquisition of associative strength with respect to a single cue on a given trial will be a function of the strength of the observed outcome minus the strength of the outcome that is expected on the basis of the predictive value of all cues present on that trial. When $\mathrm{X}$ is presented simultaneously with $\mathrm{A}$, the outcome is strongly expected on the basis of the presence of the reliable predictor of the outcome (A). Therefore, $\mathrm{X}$ acquires only a weak association with the outcome in comparison with a situation in which the target is paired with the outcome in the presence of a stimulus that has no previously established relationship with the outcome (i.e., AX + with no

R. R. Miller, rmiller@binghamton.edu 
preceding $\mathrm{A}+$ trials). The latter situation, or some similar variant, is the typical control for a blocking experiment.

Blocking has become a benchmark for associative theories. As a result, there are many accounts of blocking with mechanisms that use disparate psychological constructs, such as surprise (e.g., Rescorla \& Wagner, 1972), attention (e.g., Pearce \& Hall, 1980), and comparator processes (e.g., Miller \& Matzel, 1988). However, most associative accounts of blocking are similar in that they consistently predict blocking when the proper parameters are employed. Generally, if the blocking cue receives sufficient training to build a strong association with the outcome and the target stimulus is salient enough to avoid overshadowing in the control group, blocking should occur consistently. However, numerous recent studies have shown that blocking may be modulated by variables that are not included in the typical associative accounts. Blocking is affected by relative outcome intensity (e.g., De Houwer, Beckers, \& Glautier, 2002), test order (Lachnit et al., 1990), pretraining instruction (e.g., Mitchell \& Lovibond, 2002), pretraining with nonblocking stimuli (e.g., Beckers, De Houwer, Pineño, \& Miller, 2005), causal structure (e.g., De Houwer et al., 2002), distractor tasks (De Houwer \& Beckers, 2003), and the nature of the experimental task (e.g., Melchers, Üngör, \& Lachnit, 2005), among other factors. For the purposes of this article, we will limit the scope of the variables to include only instances in which pretraining with, or instruction about, a set of cues affects future processing of a different set of cues.

Inspired by previous studies with human subjects (e.g., Beckers et al., 2005; Mitchell \& Lovibond, 2002), Beckers, Miller, De Houwer, and Urushihara (2006, Experiment 1) conducted a conditioned barpress (BP) suppression study with rat subjects in which they examined the effect of subadditive pretraining on subsequent blocking training. Here, the phrase subadditive pretraining is used to refer to training that is designed to teach subjects that two reliable predictors of a common outcome do not summate when they are presented together. In their preparation, the cues were audiovisual stimuli (denoted by letters) and the outcome was a footshock (denoted by a "+"). Subjects that experienced subadditive pretraining $(\mathrm{C}+/ \mathrm{D}+/ \mathrm{CD}+)$ before blocking training $(\mathrm{A}+$ followed by $\mathrm{AX}+)$ exhibited less blocking than did subjects that were given an irrelevant form of pretraining $(\mathrm{C}+/ \mathrm{D}+/ \mathrm{E}+$ or $\mathrm{C}+/ \mathrm{C}+/ \mathrm{DE}+)$. That is, fear of $X$ was greater in the subjects that received subadditive pretraining followed by blocking training than it was in those that received irrelevant pretraining followed by blocking training. Similar observations in studies with human subjects were interpreted as evidence that participants show blocking only when they assume that two reliable predictors should summate when presented together. When this assumption is contradicted by subadditive pretraining, blocking is not observed when the subjects subsequently process novel cues. The observed effect of subadditive pretraining on blocking in nonhuman animals suggests that rats possess the cognitive abilities necessary to successfully apply past learning about one set of cues to a different set of cues. Although the psychological mechanism might vary between species, the effectiveness of subadditive pretraining does appear to generalize across species, at least to rats and humans.

As mentioned above, most associative theories have not accounted for fundamental changes in the way that new stimuli are processed on the basis of past experience with different stimuli. Although these theories might account for generalization of a conditioned response from a previously trained stimulus to a new stimulus (e.g., Estes, 1950; Pearce, 1994), they do not account for the generalization of an acquired rule or assumption regarding stimulus interaction or processing. Although Beckers et al. (2005) provided a framework that explains the effect of subadditive pretraining on blocking, it was somewhat unsatisfying. This account did not specify the conditions under which past learning would generalize to new cues. It should be noted here that this criticism arises from an absence of specific mechanisms to guide generalization, but does not undermine any of the fundamental assumptions made by Beckers et al. (2005). Indeed, this failing is quite understandable, because little data have been collected to guide the formulation of specific mechanisms.

The following experiments were conducted in order to identify some of the factors that constrain the generalization of subadditive pretraining. In the experiments, we investigated variables that are likely to impact generalization, on the basis of prior research. Experiments 1, 2, and 3 involved manipulations of the physical context, the retention interval, and the temporal characteristics of the CSs, respectively. All of the experiments used a conditioned BP suppression preparation with rat subjects. This experimental design was selected in order to replicate Beckers et al. (2006) and to illuminate the importance of these results for traditional associative theories, which are typically applied to nonhuman animal conditioning experiments.

\section{EXPERIMENT 1}

Experiment 1 was designed in order to address two goals. First, we sought to replicate the results of Beckers et al. (2006). Second, we investigated whether subadditive pretraining would generalize across physical contexts using rat subjects in a conditioned BP suppression preparation. There is a sizeable body of evidence that suggests that some forms of learning and memory can be context specific (e.g., Godden \& Baddeley, 1975). In most fearconditioning situations, the context does little to modulate responding to a CS (e.g., Hall \& Honey, 1989). However, with very few conditioning trials, a context shift between training and testing can attenuate conditioned responding (e.g., Hall \& Honey, 1990). Furthermore, in more complex learning paradigms, there are a number of examples of the context modulating responding in Pavlovian conditioning situations. For example, renewal from extinction (e.g., Bouton \& King, 1983), the context specificity of latent inhibition (e.g., Lovibond, Preston, \& Mackintosh, 1984), and occasion setting (e.g., Holland, 1992) all demonstrate the influence of the context on Pavlovian conditioning. In Experiment 1 (see Table 1), subjects were given either subadditive pretraining $(\mathrm{C}+/ \mathrm{D}+/ \mathrm{CD}+)$ or irrelevant pretraining in Phase $1(\mathrm{C}+/ \mathrm{C}+/ \mathrm{D}+)$. In Phases 2 
Table 1

The Design of Experiment 1

\begin{tabular}{lcccc}
\hline \multicolumn{1}{c}{ Group } & \multicolumn{1}{c}{ Phase 1 } & Phase 2 & Phase 3 & Test X \\
\hline Irrelevant-ctrl-no-shift & {$[4 \mathrm{C}+/ 4 \mathrm{C}+/ 4 \mathrm{D}+]_{1}$} & {$[12 \mathrm{~B}+]_{1}$} & {$[4 \mathrm{AX}+]_{1}$} & {$[4 \mathrm{X}-]_{3}$} \\
Irrelevant-block-no-shift & {$[4 \mathrm{C}+/ 4 \mathrm{C}+/ 4 \mathrm{D}+]_{1}$} & {$[12 \mathrm{~A}+]_{1}$} & {$[4 \mathrm{AX}+]_{1}$} & {$[4 \mathrm{X}-]_{3}$} \\
Subadditive-ctrl-no-shift & {$[4 \mathrm{C}+/ 4 \mathrm{D}+/ 4 \mathrm{CD}+]_{1}$} & {$[12 \mathrm{~B}+]_{1}$} & {$[4 \mathrm{AX}+]_{1}$} & {$[4 \mathrm{X}-]_{3}$} \\
Subadditive-block-no-shift & {$[4 \mathrm{C}+/ 4 \mathrm{D}+/ 4 \mathrm{CD}+]_{1}$} & {$[12 \mathrm{~A}+]_{1}$} & {$[4 \mathrm{AX}+]_{1}$} & {$[4 \mathrm{X}-]_{3}$} \\
Subadditive-ctrl-shift & {$[4 \mathrm{C}+/ 4 \mathrm{D}+/ 4 \mathrm{CD}+]_{2}$} & {$[12 \mathrm{~B}+]_{1}$} & {$[4 \mathrm{AX}+]_{1}$} & {$[4 \mathrm{X}-]_{3}$} \\
Subadditive-block-shift & {$[4 \mathrm{C}+/ 4 \mathrm{D}+/ 4 \mathrm{CD}+]_{2}$} & {$[12 \mathrm{~A}+]_{1}$} & {$[4 \mathrm{AX}+]_{1}$} & {$[4 \mathrm{XX}-]_{3}$} \\
\hline
\end{tabular}

Note-Numerals indicate number of trials. The "+" represents a footshock; C and D represent a buzzer and a flashing light, counterbalanced; A and B represent a Sonalert and a low tone, counterbalanced; X represents a click train; []$_{1}$ denotes that training occurred in Context $1 ;[]_{2}$ denotes that training occurred in Context 2 ; all testing occurred in Context 3 ; slashes separate interspersed trials.

and 3, subjects were given either blocking training $(\mathrm{A}+$ followed by $\mathrm{AX}+)$ or blocking control training $(\mathrm{B}+$ followed by $\mathrm{AX}+$ ). In order to study the generality of subadditive pretraining, the physical context was shifted between Phases 1 and 2 (the shift condition) for half of the subjects in the subadditive condition. The central question was whether shifting the physical context between Phases 1 and 2 would attenuate the effect of subadditive pretraining by decreasing the similarity of the two training situations. In order to conduct this experiment, three different contexts were used. Subjects in the no-shift condition received all training in the same context, whereas the context was shifted between Phases 1 and 2 for subjects in the shift condition. All baseline BP training and testing occurred in a neutral third context in order to avoid differences in fear elicited by the test context.

\section{Method}

\section{Subjects}

The subjects were 36 male (290-387 g) and 36 female (195$257 \mathrm{~g}$ ), experimentally naive, Sprague-Dawley-descended rats obtained from our own breeding colony at SUNY Binghamton. Subjects were randomly assigned to one of the six groups $(n \mathrm{~s}=12)$, counterbalanced within groups for sex. The animals were individually housed in standard hanging stainless steel wire-mesh cages in a vivarium maintained on a 16:8-h light:dark cycle. Experimental manipulations occurred near the middle portion of the light phase. The animals were allowed free access to Purina Lab Chow, whereas water availability was limited to $20 \mathrm{~min} /$ day following a progressive deprivation schedule initiated 1 week prior to the start of the study. From the time of weaning until the start of the study, all animals were handled for $30 \mathrm{sec}$, three times per week.

\section{Apparatus}

Three contexts were used in this experiment. Phases 1, 2, and 3 took place in Contexts 1 and 2, which were rectangular and truncatedV-shaped chambers, counterbalanced. All chambers were enclosed within sound-attenuating isolation chests. The V-shaped chambers were $27 \mathrm{~cm}$ long, $29.5 \mathrm{~cm}$ high, $21.5 \mathrm{~cm}$ wide at the top, and $5.5 \mathrm{~cm}$ wide at the bottom. The ceiling was clear Plexiglas, the front and back walls were black Plexiglas, and the sidewalls were stainless steel. The floor was comprised of two $27-\mathrm{cm}$-long stainless steel plates, $2 \mathrm{~cm}$ wide, with a $1.5-\mathrm{cm}$ gap between the two plates. The chamber was illuminated by a $7-\mathrm{W}$ (nominal at $120 \mathrm{VAC}$, but driven at $60 \mathrm{VAC}$ ) lightbulb, which was mounted on the inside wall of the environmental enclosure, approximately $30 \mathrm{~cm}$ from the center of the experimental chamber. The rectangular chambers each measured $24.0 \times 9.0 \times 12.5 \mathrm{~cm}(1 \times \mathrm{w} \times \mathrm{h})$. The walls and ceiling were clear
Plexiglas, and the floor comprised stainless steel rods measuring $0.5 \mathrm{~cm}$ in diameter, spaced $1.5 \mathrm{~cm}$ apart (center to center). Each chamber was dimly illuminated by a $2-\mathrm{W}$ (nominal at $120 \mathrm{VAC}$, but driven at $60 \mathrm{VAC}$ ) incandescent houselight mounted on an inside wall of the environmental chest located approximately $30 \mathrm{~cm}$ from the animal enclosure. The light intensities inside the two chambers were approximately equal due to the difference in opaqueness of the walls between the rectangular and the V-shaped chambers.

For all animals, BP training and CS testing occurred in Context 3. The 12 instances of Context 3 were square chambers, each measuring $30 \times 30 \times 27 \mathrm{~cm}(1 \times \mathrm{w} \times \mathrm{h})$. The sidewalls of the chamber were made of stainless steel sheet metal, and the front wall, back wall, and ceiling of the chamber were made of clear Plexiglas. On one metal wall of each chamber, there was an operant bar and, horizontally adjacent to it, a niche $(4.5 \times 4.0 \times 4.5 \mathrm{~cm})$ centered $3.3 \mathrm{~cm}$ above the floor. A solenoid valve could deliver $0.04 \mathrm{ml}$ of water into a cup on the bottom of the niche. The floor was constructed of 0.3 -cm-diameter rods, spaced $1.3 \mathrm{~cm}$ center to center. Each chamber was housed in an environmental isolation chest, which was dimly illuminated by a houselight (1.12-W, No. 1820 incandescent bulb) mounted on one wall of the experimental chamber.

All chambers were equipped with three $45-\Omega$ speakers on the inside walls of the isolation chests that could deliver a low-frequency complex tone ( 300 and $320 \mathrm{~Hz}) 8 \mathrm{~dB}$ (C-scale) above background ( $76 \mathrm{~dB}$, produced mainly by a ventilation fan), a white noise $8 \mathrm{~dB}(\mathrm{C})$ above background, or a click train $(6 / \mathrm{sec}) 8 \mathrm{~dB}(\mathrm{C})$ above background. A visual stimulus that consisted of a flashing light $(0.5 \mathrm{sec}$ on, $0.5 \mathrm{sec}$ off) could also be presented. The flashing light cue was provided by a $25-\mathrm{W}$ bulb (nominal at $120 \mathrm{VAC}$, driven at $85 \mathrm{VAC}$ ) in the rectangular chambers and a $100-\mathrm{W}$ bulb (nominal at $120 \mathrm{VAC}$, driven at $85 \mathrm{VAC}$ ) in the V-shaped chambers. The bulbs were mounted on an inside wall of the environmental chest, approximately $30 \mathrm{~cm}$ from the center of the experimental chamber. Additionally, a Sonalert (Mallory Sonalert Products, Indianapolis) mounted on each environmental chest was able to deliver a high-frequency $(1900-\mathrm{Hz})$ tone $8 \mathrm{~dB}(\mathrm{C})$ above background, and a buzzer was able to deliver a buzzing sound $8 \mathrm{~dB}(\mathrm{C})$ above background. The $0.7-\mathrm{mA}$ footshock, which served as the US, could be delivered through the chamber floors. In this experiment, all CSs were $30 \mathrm{sec}$ in duration, and the footshock US was $0.5 \mathrm{sec}$ in duration. CSs C and D were the buzzer and the flashing light, counterbalanced. CSs A and B were the Sonalert and the low tone, counterbalanced. CS X was the click train.

\section{Procedure}

Baseline training. On Days $1-5$, BP training was conducted in daily 1-h sessions in Context 3. Animals were trained to press an operant bar for water in 0.04-ml servings; to make water delivery more conspicuous, a 0.5 -sec white noise was presented simultaneously with the delivery of water reinforcement. On Days 1 and 2, a fixed-time 120 -sec schedule of signaled noncontingent water delivery operated concurrently with a continuous reinforcement (CRF) 
schedule for barpressing. On Day 3, subjects were trained on the $\mathrm{CRF}$ schedule alone. Animals that made fewer than 50 BPs received 30 min of hand shaping. On Days 4 and 5, subjects were trained on a VI-20-sec schedule.

Phase 1: Subadditivity pretraining. On Days 6-9, blocking and control animals in the subadditive condition were exposed daily to one pairing of each of two elemental cues $(C$ and $D)$ with the footshock US as well as one pairing of the CD compound with the US. Termination of the US coincided with the termination of the cue. The irrelevant condition animals were exposed daily to two pairings of $\mathrm{C}$ with the US and one pairing of D with the US. On Days 6 and 8, the order of trials was C, D, CD (or C, C, D); on Days 7 and 9, the order of trials was C, CD, D (or C, D, C). CSs were presented 15, 24, and $45 \mathrm{~min}$ into the session on Days 6 and 7. On Days 8 and 9, CSs were presented at 9, 30, and 45 min into the session. The pretraining sessions occurred in Context 1 for subjects in the no-shift condition (including all subjects in the irrelevant condition) and in Context 2 for subjects in the shift condition. (See Table 1.)

Phase 2: Elemental training. On Days 10-12 in Context 1, the blocking animals in all conditions were exposed daily to four pairings of A with the US, with A and the US coterminating. The control animals received similar pairings of cue B with the US. Pairings were presented 18, 24, 36, and 54 min into the session on all days.

Phase 3: Compound training. On Day 13 in Context 1, all animals received four pairings of the compounded presentation of A and $\mathrm{X}$ with the US, with parameters similar to those for the previous phase.

Baseline recovery. In Context 3, on Days 14-16, all animals were retrained to barpress for water in 0.04-ml servings on the VI20 -sec schedule. No experimental stimuli were presented.

Test. On Day 17 in Context 3, all subjects were tested on X. The $\mathrm{X}$ stimulus was presented four times, for $30 \mathrm{sec}$ each, 6, 12, 18, and 24 min into the session. The number of BP responses during $X$ was compared with half the number of BPs during a 1-min period immediately preceding the onset of X. Due to a mechanical failure, 1 rat from the irrelevant-block-no-shift group was eliminated from the experiment. To reduce variability, but to preserve sensitivity to changes that occur with repeated testing, the data were blocked so that Test Trials 1 and 2 were pooled, as were Test Trials 3 and 4 . Thus, two suppression ratios were analyzed for each subject. The suppression ratios for each subject were calculated by dividing the total number of BPs made during the first two or last two CS presentations by the sum of that number plus half the total number of BPs made during the 60 -sec intervals that immediately preceded each 30 -sec CS [i.e., $\left.\mathrm{BP}_{\mathrm{CS}} /\left(\mathrm{BP}_{\mathrm{CS}}+0.5 \mathrm{BP}_{\text {pre-CS }}\right)\right]$. With this measure, a ratio of $.50 \mathrm{de}-$ notes no suppression, whereas a ratio of 0 denotes complete suppression in the presence of the CS. We used 60 -sec baseline measures as opposed to $30-\mathrm{sec}$ measures, on the basis of the assumption that a larger time sample would better assess average rates of responding. In this and the following experiments, subjects that did not register any pre-CS responses in either of the two-trial blocks were eliminated from the data analysis because no suppression ratio could be calculated for that block. In Experiment 1, no animal was eliminated according to this criterion.

\section{Results and Discussion}

The data from the subjects in the no-shift condition are displayed in Figure 1, and the data from the subjects in the subadditive condition are displayed in Figure 2. As can be seen in Figure 1, the suppression ratios indicate a blocking effect when irrelevant pretraining was administered, but less of a blocking effect when subadditive pretraining (Phase 1) was given in the same context as that for the blocking training (Phases 2 and 3). Furthermore, Figure 2 shows that the effect of subadditive pretraining was weakened when it was administered in a different context than was the blocking training. This suggests that subadditive training does not

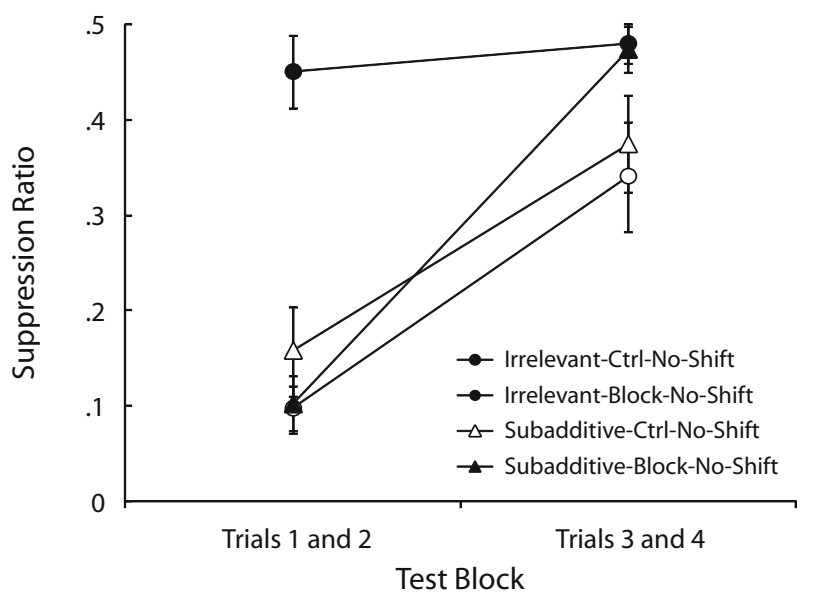

Figure 1. Suppression ratios in response to $X$ in each of two blocks of test trials in Experiment 1 for subjects that received all training in the same context. Lower ratios indicate less barpressing, which is indicative of better conditioned responding to the fear-invoking stimulus. Therefore, higher scores for blocking groups than for control groups are indicative of blocking. Error brackets denote the standard error of the mean for each group. See Table 1 for procedural details.

fully generalize to a new context. These observations were supported by the following statistical analyses.

Before we analyzed the responding to $\mathrm{X}$, the pre-CS leverpress rates from the first test trial were analyzed to determine whether there were any differences between groups in baseline rates of leverpressing before any CS presentations. Subjects in the irrelevant-no-shift condition registered $25.83(S E=4.78)$ and $24.18(S E=2.65)$ mean leverpresses in the ctrl and block groups, respectively. Subjects in the subadditive-no-shift condition registered 21.00 $(S E=2.67)$ and $20.75(S E=3.26)$ mean leverpresses

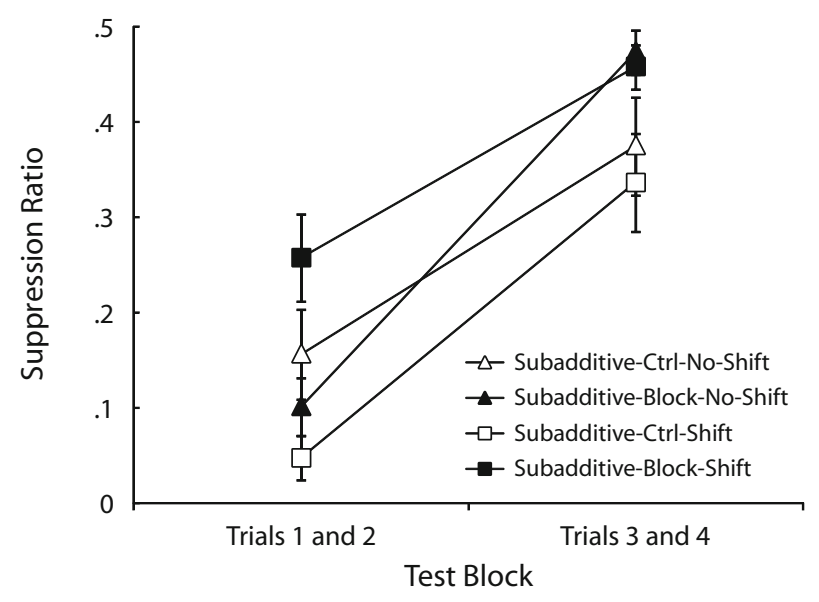

Figure 2. Suppression ratios in response to $X$ in each of two blocks of test trials in Experiment 1 for subjects that received subadditive pretraining. Lower ratios indicate less barpressing, which is indicative of better conditioned responding to the fearinvoking stimulus. Therefore, higher scores for blocking groups than for control groups are indicative of blocking. Error brackets denote the standard error of the mean for each group. See Table 1 for procedural details. 
in the ctrl and block groups, respectively. Subjects in the subadditive-shift condition registered $20.92(S E=3.51)$ and $21.75(S E=3.26)$ mean leverpresses in the ctrl and block groups, respectively. A 3 (pretraining: subadditive vs. subadditive shift vs. irrelevant) $\times 2$ (training: block vs. ctrl) ANOVA revealed no significant main effects or interaction $(p \mathrm{~s}>.39)$.

An ANOVA of the suppression ratios now including test trial block as an additional variable revealed an effect of training $[F(1,65)=26.81, p<.05]$, an effect of test trial $[F(1,65)=158.82, p<.05]$, interactions between pretraining and training $[F(2,65)=5.53, p<.05]$ and between pretraining and test trial $[F(2,65)=6.77, p<$ $.05]$, and a three-way interaction $[F(2,65)=9.14, p<$ $.05]$. In order to determine the source of the interactions, two $2 \times 2 \times 2$ ANOVAs were used to analyze the CS data. The first ANOVA determined whether Experiment 1 replicated the results of Beckers et al. (2006). A 2 (pretraining: subadditive vs. irrelevant) $\times 2$ (training: block vs. ctrl) $\times 2$ (test block: 1 and 2 vs. 3 and 4) ANOVA of the data from subjects in the no-shift condition revealed a main effect of training, a main effect of test block, an interaction between test block and pretraining (all $p \mathrm{~s}<.01$ ) and, most important, an interaction between pretraining and training $[F(1,43)=9.54, p<.01]$, replicating Beckers et al.'s (2006) previous finding. A planned comparison of the irrelevant blocking no-shift group with the irrelevant control no-shift group indicated a blocking effect when irrelevant pretraining preceded blocking training $[F(1,43)=21.41, p<.01]$. No significant blocking effect was observed when the subjects experienced subadditive pretraining $[F(1,43)=0.18, p=.67]$.

A second 2 (context: shift vs. no shift) $\times 2$ (training: block vs. ctrl) $\times 2$ (test block: 1 and 2 vs. 3 and 4 ) ANOVA of the data from subjects in the subadditive condition was conducted to determine whether the subadditive pretraining was equally effective when administered in a different context from that of the blocking training. The analysis revealed a main effect of training, a main effect of test block, and a three-way interaction between training, context, and test block (all $p \mathrm{~s}<.05$ ). Also, an interaction between context and training indicated that blocking was more robust when the context of pretraining differed from the context of blocking training $[F(1,44)=45.57, p<$ $.05]$. A planned comparison of the subadditive-blockshift and subadditive-ctrl-shift groups revealed a blocking effect $[F(1,44)=12.08, p<.01]$. However, a direct comparison between the groups that received blocking training (subadditive-block-shift and subadditive-blockno-shift) revealed no significant difference in responding $[F(1,44)=2.19, p=.15]$. Figure 2 shows that a difference between these groups might have occurred in the first block of test trials, but not in the second. This observation is supported by the significant three-way interaction. A comparison between these groups using only the data from Test Trials 1 and 2 did reveal weaker suppression in the subadditive-block-shift group than in the subadditive-block-no-shift group $[F(1,44)=7.78, p<$ $.01]$. This difference was not significant on Trials 3 and 4 $[F(1,44)=0.07, p=.80]$. The absence of a difference in Trials 3 and 4 was likely due to extinction, which appeared to be more rapid in the subadditive-block-no-shift group relative to the blocking control groups. This rapid extinction could indicate that the subadditive pretraining does not completely eliminate the influence of A on X. Thus, we can conclude that although the effect was observed only in the first two test trials, the physical context change modulated the effect of subadditive pretraining on blocking.

To interpret the results of Experiment 1, as well as those of the subsequent experiments, note that the effect of subadditive pretraining takes the form of a double negative. The target cue's association with the US drives conditioned responding. The influence of the blocking cue impairs that responding. The subadditive pretraining impairs the effectiveness of the blocking cue, thus augmenting responding to $\mathrm{X}$. Therefore, a reduction in responding to $\mathrm{X}$ in the shift condition could be due to reduced excitatory strength, to enhanced blocking, or to an attenuation of the influence of subadditive pretraining. Considered in the framework of Beckers et al.'s (2006) interpretation of the subadditive pretraining effect, the data suggest that the learning imbued by subadditive pretraining is at least partially specific to the context in which pretraining was administered. Testing occurred in a neutral context for all subjects; thus, subadditive pretraining generalized well to a novel test context, but was limited in its effectiveness if it occurred outside of the context of blocking treatment.

Other potential interpretations include the possibility that blocking was facilitated by the context shift between Phases 1 and 2, or that responding to $\mathrm{X}$ was more directly weakened by the context shift. We see no obvious and principled reason to have expected enhanced blocking in the present situation (or the subsequent experiments), and the observed blocking effect in the irrelevant condition was robust to the point that it would be difficult to enhance. However, it is possible that the present study does not demonstrate a limitation in the generalization of subadditive pretraining or an enhancement of blocking, but instead shows a limitation in the generalization of simple conditioned responding. That is, subadditive pretraining in this situation might have resulted in greater conditioning of the $\mathrm{C}$ and $\mathrm{D}$ stimuli than did irrelevant pretraining. For example, a straightforward application of the RescorlaWagner (1972) model would suggest that subadditive pretraining would result in nearly equal conditioning of $\mathrm{C}$ and $\mathrm{D}$, whereas irrelevant pretraining would result in slightly stronger conditioning of $\mathrm{C}$, but much weaker conditioning of D. Thus, the combined associative strengths of C and D would be higher after subadditive pretraining. If one assumes robust generalization from both $\mathrm{C}$ and $\mathrm{D}$ to $\mathrm{X}$, the difference between the subadditive and irrelevant pretraining could be due to generalization of simple excitatory conditioning, which was attenuated when the context was shifted between pretraining and blocking training.

There is good reason, however, to question the simple generalization account described above. The effect of subadditive pretraining was profound in the blocking condition, which suggests that generalization would need to be far beyond what is typically observed in our laboratory. On 
Trials 1 and 2, subadditive pretraining virtually eliminated a blocking effect that was, itself, almost complete. Furthermore, this generalization was inexplicably absent in the control condition, both on Trials 1 and 2 and on Trials 3 and 4, in which responding was substantially weaker. If anything, the control subjects appeared to show the opposite tendency.

\section{EXPERIMENT 2}

Experiment 1 showed that subadditive pretraining can attenuate blocking, replicating one result of Beckers et al. (2006). Furthermore, the effect of subadditive pretraining was at least partially context specific; blocking was more apparent when subjects experienced subadditive pretraining and blocking training in different contexts rather than in the same context.

In Experiment 2, we investigated the generalization of subadditive pretraining over time. Although Pavlovian conditioning tends to be persistent over a long retention interval, in many situations the passage of time can produce changes in conditioned responding that are very similar to those produced by a shift in the physical context (for a review, see Bouton, 1993). Bouton suggested that the passage of time might alter internal and external cues, which would result in a gradual change in the context. Like the physical context manipulation in Experiment 1, a long retention interval might reduce generalization of subadditive pretraining, perhaps by producing a temporal context shift. In Experiment 2, the temporal interval between Phases 1 and 2 was manipulated. Thus, the design of Experiment 2 was very similar to the design of Experiment 1 , except that instead of a physical context shift, some subjects were given a long (21-day) retention interval between Phases 1 and 2, whereas other subjects were given a short (1-day) retention interval equivalent to that used in all groups in Experiment 1 (see Table 2). With the short retention interval, we expected results similar to those observed in the no-shift condition of Experiment 1, but with the long retention interval, it was possible that blocking would be more apparent (denoted by a numerically larger suppression ratio in the blocking group). Also, because we successfully replicated the results of Beckers et al. (2006) in Experiment 1 and there was no reason to anticipate an effect of this sort of retention interval on blocking, the irrelevant pretraining control groups were not included in Experiment 2. Finally, there was no reason to use more than one context in this experiment, so all training and

Table 2

The Design of Experiment 2

\begin{tabular}{llcccc}
\hline \multicolumn{5}{c}{ Group } & \multicolumn{5}{c}{ Interval } \\
Phase 1 & (Days) & Phase 2 & Phase 3 & Test \\
\hline Ctrl-short & $4 \mathrm{C}+/ 4 \mathrm{D}+/ 4 \mathrm{CD}+$ & 1 & $12 \mathrm{~B}+$ & $4 \mathrm{AX}+$ & $4 \mathrm{X}-$ \\
Block-short & $4 \mathrm{C}+/ 4 \mathrm{D}+/ 4 \mathrm{CD}+$ & 1 & $12 \mathrm{~A}+$ & $4 \mathrm{AX}+$ & $4 \mathrm{X}-$ \\
Ctrl-long & $4 \mathrm{C}+/ 4 \mathrm{D}+/ 4 \mathrm{CD}+$ & 21 & $12 \mathrm{~B}+$ & $4 \mathrm{AX}+$ & $4 \mathrm{X}^{-}$ \\
Block-long & $4 \mathrm{C}+/ 4 \mathrm{D}+/ 4 \mathrm{CD}+$ & 21 & $12 \mathrm{~A}+$ & $4 \mathrm{AX}+$ & $4 \mathrm{X}-$ \\
\hline
\end{tabular}

Note-Numerals indicate number of trials. The "+" represents a footshock; C and D represent a buzzer and flashing light, counterbalanced; A and $\mathrm{B}$ represent a Sonalert and a low tone, counterbalanced; $\mathrm{X}$ represents a click train; slashes separate interspersed trials. testing sessions were administered in one context (which was the same as Context 3 from Experiment 1).

\section{Method}

\section{Subjects}

The subjects were 24 male (225-290 g) and 24 female (165-215 g), experimentally naive, Sprague-Dawley-descended rats obtained from our own breeding colony at SUNY Binghamton. Subjects were randomly assigned to one of the four groups $(n s=12)$, counterbalanced within groups for sex. The animals were individually housed, water deprived, and handled as were those in Experiment 1.

\section{Apparatus}

All experimental treatments occurred in Context 3 as described in Experiment 1. The stimuli were identical to those used in Experiment 1 . The only exception was that the bars were retracted during all of the training phases (Phases 1-3).

\section{Procedure}

Baseline training. On Days 1-5, BP training was conducted as it was in Experiment 1. After Day 5, the bars in all of the chambers were retracted.

Phase 1: Subadditivity pretraining. The subjects in the long condition received Phase 1 training on the day after baseline BP training ended and 20 days before the subjects in the short condition. On Days 6-9, blocking and control animals in the long condition were exposed daily to one pairing of each of two elemental cues (C and D) with the US, as well as to one pairing of the $\mathrm{CD}$ compound with the US. Termination of the US coincided with the termination of the cue. On Days 6 and 8, the order of trials was C, D, CD; on Days 7 and 9 , the order of trials was C, CD, D. CSs were presented 15,24 , and 45 min into the session on Days 6 and 7. On Days 8 and 9, CSs were presented at 9, 30, and 45 min into the session. After Day 9 , the subjects in the long condition remained in their home cages until Day 30 and were handled thrice weekly for $30 \mathrm{sec}$. The subjects in the short condition remained in their home cages after baseline BP training, except for thrice weekly handling, until they began Phase 1 training on Day 26. The sessions were identical to those administered in the long condition.

Phase 2: Elemental training. On Days 30-32, the animals in the blocking condition were exposed daily to four pairings of A with the US, with A and the US coterminating. The control animals received similar pairings of cue B with the US. Pairings were presented 18, 24,36 , and 54 min into the session on all days.

Phase 3: Compound training. On Day 33, all animals received four pairings of the compounded presentation of $\mathrm{A}$ and $\mathrm{X}$ with the US, with parameters similar to those for the previous phase.

Baseline recovery. On Days 34-36, the bars were extended and all animals were retrained to barpress for water in 0.04-ml servings on the VI-20-sec schedule. No experimental stimuli were presented.

Tests. On Day 37, all subjects were tested on X. The X stimulus was presented four times, for 30 sec each at 6, 12, 18, and 24 min into the session. The number of BP responses during $\mathrm{X}$ was compared with half the number of BPs during a 1-min period immediately preceding onset of X. As in Experiment 1, the data were blocked to reduce variability but still capture any changes in responding that occurred through repeated testing. Because of a mechanical failure, data from 2 rats (1 each from the ctrl-long and block-short groups) were eliminated. Another rat from the ctrl-short group did not register any BPs immediately before the second, third, and fourth CS presentations, presumably because of extreme fear of the test context. Therefore, this subject's data were excluded from the analyses.

\section{Results and Discussion}

The data from Experiment 2 are presented in Figure 3. In both blocks of test trials, blocking appeared to be more robust in the long condition. This observation is supported 
by the following statistical analyses. The mean numbers of pre-CS leverpresses were $27.55(S E=3.27), 28.27(S E=$ 4.99), $26.00(S E=4.71)$, and $31.18(S E=4.38)$ for the block-short, ctrl-short, block-long, and ctrl-long groups, respectively. A 2 (retention interval: short vs. long) $\times 2$ (training: block vs. ctrl) ANOVA revealed no significant main effects or interactions (all $p \mathrm{~s}>.50$ ). A 2 (retention interval: short vs. long) $\times 2$ (training: block vs. ctrl) $\times 2$ (test block: 1 and 2 vs. 3 and 4) ANOVA on the suppression ratios indicated an interaction between retention interval and training $[F(1,41)=4.39, p<.05]$ but no other significant effects $(p \mathrm{~s}>.10)$. A planned comparison of the block-short and ctrl-short groups showed no reliable effect of blocking $[F(1,41)=0.10, p=.76]$. However, the block-long group did show weaker responding than the ctrl-long group $[F(1,41)=7.19, p<.05]$. This suggests that blocking was apparent when a long retention interval separated subadditive pretraining and blocking training. A direct comparison of responding in the two blocking groups supported this conclusion; the block-long group showed weaker responding than did the block-short group $[F(1,41)=5.19, p<.05]$.

The results of Experiment 2 suggest that subadditive pretraining is less effective if it is administered long before blocking training. If this is considered as a limit on the generalization of subadditive pretraining, then it stands in contrast to what is widely known about the generalization of simple conditioned responding. Many studies have indicated that generalization gradients flatten as time passes, causing more stimuli to control behavior with the same efficacy as the original target cue (for a review, see Riccio, Richardson, \& Ebner, 1984). To the extent that this is a general phenomenon, the generalization of subadditive pretraining appears to be dissociated from simple associative generalization. However, Riccio and his colleagues (Riccio, Ackil, \& Burch-Vernon, 1992;

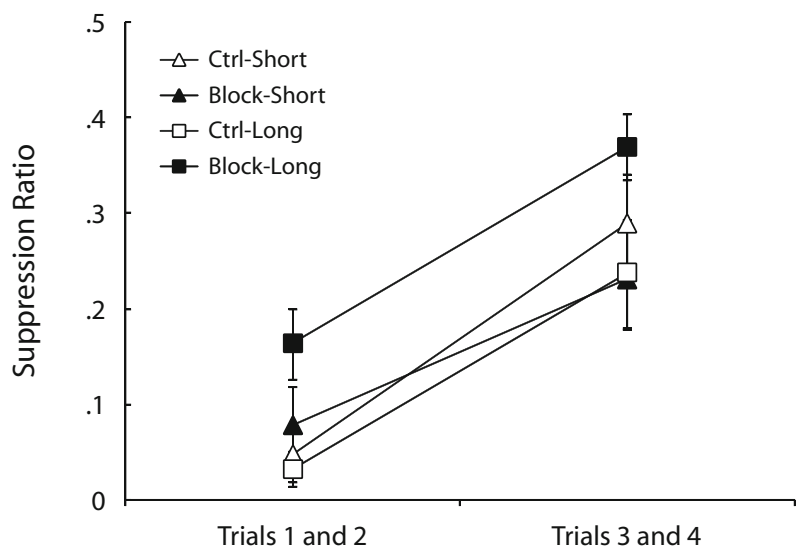

Test Block

Figure 3. Suppression ratios in response to $X$ in each of two blocks of test trials in Experiment 2 for all subjects. Lower ratios indicate less barpressing, which is indicative of better conditioned responding to the fear-invoking stimulus. Therefore, higher scores for blocking groups than for control groups are indicative of blocking. Error brackets denote the standard error of the mean for each group. See Table 2 for procedural details.
Riccio et al., 1984) have suggested that the flattening of generalization gradients could be caused by a process that might also result in reduced generalization of subadditive pretraining. They viewed the flattening of generalization gradients as a consequence of forgetting ${ }^{1}$ stimulus attributes. According to this notion, generalization increases with time because subjects forget about characteristics of the target stimulus and fail to discriminate between the target and other, nontarget stimuli. If one assumes that subadditive pretraining teaches subjects about the way that a stimulus interacts with other stimuli, then they may forget this sort of stimulus attribute long before they forget a basic CS-US association. Alternatively stated, subadditivity may be encoded as a stimulus property that becomes less retrievable with time.

\section{EXPERIMENT 3}

Experiments 1 and 2 demonstrated that the effect of subadditive pretraining does not generalize fully to a new context, or across a long retention interval. Experiment 3 was designed to determine whether CS similarity is necessary for subadditive pretraining to be effective. In this experiment, similarity was manipulated by altering the temporal characteristics of the CSs (e.g., Escobar \& Miller, 2003). As in Experiment 2, all of the subjects were given subadditive pretraining. However, the temporal characteristics of the CSs between pretraining (Phase 1) and blocking training (Phases 2 and 3 ) were manipulated by varying the CS duration. In order to manipulate the temporal characteristics of the CSs, the CS durations were either long $(60 \mathrm{sec})$ or short $(15 \mathrm{sec})$. For counterbalancing purposes, half of the subjects in the same condition experienced CS durations that were all long, whereas the other half experienced CS durations that were all short. Half of the subjects in the different (diff) condition received short CSs in Phase 1, and long CSs in Phases 2 and 3. The other half received the opposite treatment. Testing was conducted in all groups with a 30 -sec CS. If the efficacy of pretraining is constrained by similarity of the temporal characteristics of the stimuli, more blocking should be detected in the diff condition than in the same condition (revealed as a numerically larger suppression ratio).

Method
Subjects
The subjects were 24 male $(245-300 \mathrm{~g})$ and 24 female $(180-$
$213 \mathrm{~g})$, experimentally naive, Sprague-Dawley-descended rats
obtained from our own breeding colony at SUNY Binghamton.
Subjects were randomly assigned to one of the four groups, each
of which had two subgroups $(n \mathrm{~s}=6)$, counterbalanced within sub-
groups for sex. The animals were individually housed, deprived, and
handled as were those in Experiments 1 and 2 .

\section{Apparatus}

The context of training and testing was identical to that used in Experiment 2.

\section{Procedure}

The experiment involved 1-h sessions on each day. Animals were run in four squads of 12 rats each, counterbalanced with respect to group. 
Baseline training. On Days 1-5, BP shaping was conducted as it was in Experiments 1 and 2. As in Experiment 2, the bars in all of the chambers were retracted after Day 5.

Phase 1: Subadditivity pretraining. Every group was split into two subgroups. The subgroups received different durations of the CSs $(15$ or $60 \mathrm{sec})$ but were otherwise identical. On Days 6-9, blocking and control animals were exposed daily to one pairing of each of two elemental cues (C and D) with the US, as well as to one pairing of the CD compound with the US. Termination of the US coincided with the termination of the cue. On Days 6 and 8, the order of trials was $\mathrm{C}, \mathrm{D}, \mathrm{CD}$; on Days 7 and 9, the order of trials was C, CD, D. US onset occurred at 16, 25, and 46 min into the session on Days 6 and 7 and 10,31 , and 46 min into the session on Days 8 and 9 .

Phase 2: Elemental training. In Phases 2 and 3, subjects in the same condition were trained with CS durations that were identical to those used in Phase 1. The CS durations for subjects in the diff condition were switched so that subjects that experienced long CSs in Phase 1 experienced short CSs in Phases 2 and 3; and diff subjects that experienced short CSs in Phase 1 experienced long CSs in Phases 2 and 3. On Days 10-12, the animals in the blocking condition were exposed daily to four pairings of A with the US, with A and the US coterminating. The control animals received similar pairings of cue B with the US. US onsets occurred 19, 25, 37, and 55 min into the session on all days.

Phase 3: Compound training. On Day 13, all animals received four pairings of the compounded presentation of $\mathrm{A}$ and $\mathrm{X}$ with the US, with parameters similar to those in the previous phase.

Baseline recovery. On Days 14-16, the bars were extended, and all animals were retrained to barpress for water in 0.04-ml servings on the VI-20-sec schedule. No experimental stimuli were presented.

Test. On Day 17, all subjects were tested on X. The X stimulus was presented four times, for $30 \mathrm{sec}$ each, $6,12,18$, and 24 min into the session. The number of $\mathrm{BP}$ responses during $\mathrm{X}$ was compared with half the number of BPs during a 1-min period immediately preceding onset of X. Two rats (from the ctrl-same and block-same groups) failed to register any BPs after the initial test trial. Because no suppression ratio could be computed for these rats, their data were eliminated from all of the following analyses.

\section{Results and Discussion}

The blocked suppression ratios are displayed in Figure 4. On the first block of test trials, suppression was weakest in the block-diff group, which suggests that blocking occurred when the pretraining stimuli had temporal characteristics that differed from those of the stimuli used for blocking training. There was no apparent blocking effect in the block-same group. On the second block of test trials, suppression in the block-same group did decrease, so that it was as weak as that observed in the block-diff group. These observations were supported by the following statistical analyses.

The mean numbers of pre-CS leverpresses were 19.00 $(S E=2.19), 25.00(S E=5.81), 22.83(S E=2.40)$, and $22.58(S E=3.08)$ for the block-same, ctrl-same, blockdiff, and ctrl-diff groups, respectively. A 2 (retention interval: short vs. long) $\times 2$ (training: block vs. ctrl) ANOVA revealed no significant main effects or interaction ( $p$ s $>$ .39). A 2 (duration: same vs. diff) $\times 2$ (training: block vs. ctrl) $\times 2$ (test block: 1 and 2 vs. 3 and 4 ) ANOVA on the suppression ratios revealed an effect of training, an effect of test block, and an interaction between training and test block $(p$ s $<.01)$. Also, there was an interaction between duration and training $[F(1,42)=7.11, p<.05]$. No other main effects or interactions were detected $(p \mathrm{~s}>$

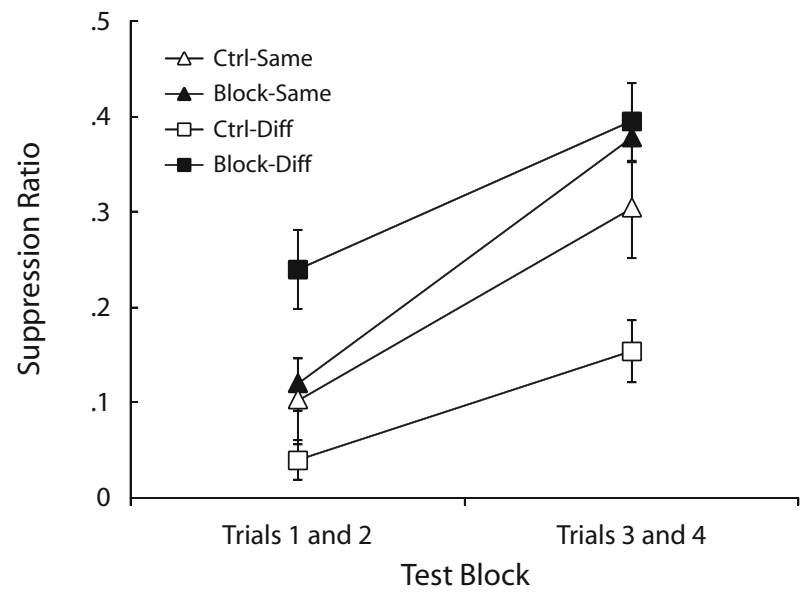

Figure 4. Suppression ratios in response to $X$ in each of two blocks of test trials in Experiment 3 for all subjects. Lower ratios indicate less barpressing, which is indicative of better conditioned responding to the fear-invoking stimulus. Therefore, higher scores for blocking groups than for control groups are indicative of blocking. Error brackets denote the standard error of the mean for each group.

.15). A planned comparison of the ctrl-same and blocksame groups revealed no blocking effect $[F(1,42)=0.96$, $p=.33]$, which demonstrates the effectiveness of subadditive pretraining also observed in Experiments 1 and 2. A comparison of the ctrl-diff and block-diff groups indicated that blocking was observed when the pretraining stimuli had different temporal characteristics than did the stimuli used for blocking training $[F(1,42)=23.79, p<$ .01]. However, a direct comparison between the blockdiff and the block-same groups revealed no significant difference in responding $[F(1,42)=2.21, p=.15]$. Similar to the results of Experiment 1, inspection of Figure 4 shows that a difference between these groups might have occurred in the first block of test trials, but not in the second. A comparison between these groups using only the data from Test Trials 1 and 2 did reveal significantly weaker responding in the block-diff group relative to the block-same group $[F(1,42)=6.02, p<.05]$. This result is similar to that observed in Experiment 1 in that extinction seemingly obscured the difference in the last two test trials. This result suggests that although the manipulation of the temporal characteristics of the stimuli might not be as effective as the retention interval employed in Experiment 2, the manipulation does impact the generalization of subadditive pretraining.

The results of Experiment 3 are consistent with those of Experiments 1 and 2 in that a change in the training conditions between pretraining and blocking training reduced the effectiveness of subadditive pretraining. However, Experiment 3 differs from the other experiments in that there appeared to be a difference between the control groups. A comparison showed generally weaker suppression in the ctrl-same group than in the ctrl-diff group $[F(1,42)=$ $5.22, p<.05]$. Unfortunately, it is difficult to determine why this occurred. It is probably not due to a difference 
in generalization of conditioned responding from $\mathrm{C}$ and $\mathrm{D}$ to $\mathrm{X}$, because the mean difference is opposite to what might be expected (i.e., more generalized fear in the same condition). It is possible that the shift in duration between Phases 1 and 2 in the shift condition enhanced the perceived similarity of $\mathrm{X}$ and $\mathrm{B}$. This could have resulted in an enhancement of the associative strength of $X$ based on generalization from B, which would have manifested as weaker responding to $\mathrm{X}$ in the ctrl-same condition than in the ctrl-diff condition. A more theory-driven explanation of this effect is discussed in the General Discussion section, but it remains unclear as to why this difference is only apparent in Experiment 3.

\section{GENERAL DISCUSSION}

The experiments reported here demonstrate that subadditive pretraining with a certain set of cues will reduce blocking between novel cues if the pretraining and blocking training are sufficiently similar. In Experiment 1, subadditive pretraining reduced blocking when the physical context used for pretraining was the same as that used for blocking training. When subadditive pretraining occurred in a different context, blocking was more readily apparent. In Experiment 2, a long retention interval interposed between pretraining and blocking training revealed blocking in subjects that experienced subadditive pretraining. Experiment 3 showed that the temporal characteristics of CSs are also important for the generalization of subadditive pretraining. Blocking was more readily apparent when the pretraining CSs had different temporal characteristics than did the blocking training CSs. Considering all of these results, it seems clear that the generalization of subadditive pretraining is determined by a number of factors that relate the pretraining situation to the blocking situation.

The present series of experiments was not designed to discriminate among theories; however, two potential accounts of the effect of subadditive pretraining will be entertained here. One account of the present data follows from research indicating that cues can be processed configurally or elementally on the basis of past experience with similar cues (for a review, see Melchers, Shanks, \& Lachnit, 2008). Specifically, Williams, Sagness, and McPhee (1994) observed that blocking in human subjects was only apparent in their preparation if the participants were previously given training that biased them toward elemental processing. If cues are processed elementally, blocking should be strong, because the presence of the reliable predictor (A) makes the target stimulus (X) redundant in terms of predictive information. In contrast, if the cues are processed configurally, the AX cue compound will be represented as a unique stimulus. Configural processing hinders the observance of blocking in two ways. First, A does not predict the outcome when AX is presented, because $\mathrm{AX}$ is represented as a unique stimulus (e.g., Livesey \& Boakes, 2004). Second, strong configuring would cause weak responding to $X$ when it is presented without $\mathrm{A}$, due to generalization decrement. That is, relatively weak responding would be observed in the typical blocking control group $(\mathrm{AX}+$ with no preceding $\mathrm{A}+$ training), which would generally reduce the sensitivity of the experimental design (e.g., Pellón \& Montaño, 1990). Williams et al. indicated that configural and elemental processing can be biased by instructions or past experience with similar stimuli. Applied to the present results, however, it is unclear as to whether subadditive pretraining resulted in configural processing. There is evidence of weak responding in the ctrl-same group of Experiment 3, but this is drawn from a comparison of two groups that both received subadditive pretraining. In Experiment 1, there was no apparent difference between the subadditive and irrelevant control groups. As such, evidence supportive of configural processing was inconsistent and absent in the situation in which subadditive pretraining had its most obvious effect on blocking. Furthermore, Williams and Braker $(1999,2002)$ referred to the type of subadditive pretraining that we gave our subjects as elemental pretraining. Elemental pretraining should, if anything, enhance blocking by reducing configuring, which is not what was observed here. In addition, Williams and Braker (2002) observed an effect of configural pretraining only in human subjects, and failed to bias rat subjects toward configural processing by using configural pretraining. The present data might stand in contrast to their result, but it would be in agreement with Alvarado and Rudy's (1992) observation that pretraining can bias configural processing in rats. However, the inconsistent evidence of configuration in the blocking control groups in Experiment 1 fails to provide unambiguous support for that interpretation.

An alternative account of the effect of subadditive pretraining observed here comes from the inferential view of blocking. The inferential view of blocking (e.g., De Houwer, Vandorpe, \& Beckers, 2005) suggests that blocking occurs because subjects assume that two reliable causes of an effect should summate (i.e., produce a more robust outcome) when presented together. Applied to the present experiments, subjects assume that two cues (e.g., A and $\mathrm{X}$ ) that are reliable causes of an outcome (shock) should have additive effects when they are presented together. In blocking $(\mathrm{A}+$ followed by $\mathrm{AX}+)$, when the AX compound is presented, the subjects infer that $\mathrm{X}$ does not cause the shock. This occurs because $\mathrm{X}$ contributed nothing to the experienced shock that was not already expected on the basis of the presence of A. There are two important implications of this account. First, it posits that blocking is a result of effortful cognitive processing on the part of the organism (De Houwer \& Beckers, 2003). Second, it implies that the fundamental assumption that underlies blocking can be altered. If subjects are informed that two reliable predictors of an outcome do not produce a more robust outcome when they are presented together either through pretraining with different cues (e.g., Beckers et al., 2006) or through instructions before blocking training (e.g., Mitchell \& Lovibond, 2002), the assumption of additivity is disregarded. The inferential view suggests that the subjects assumed additivity by default in the present experiments, which explains why robust blocking was observed in Experiment 1 after irrelevant pretraining. Subadditive pretraining $(\mathrm{C}+/ \mathrm{D}+/ \mathrm{CD}+)$ explicitly contradicted this a priori assumption of additivity. The subjects 
were taught that two reliable predictors of the outcome can produce the same outcome when they are presented together. According to the inferential view, this training should alter the assumption of additivity so that subjects would no longer expect summation of A and $\mathrm{X}$ when they are presented later in training. Although this account explains the attenuation of blocking, it does not explain the incomplete generalization of subadditive pretraining observed in Experiments 1-3.

Both of the aforementioned theories can explain the detrimental effect of subadditive pretraining on blocking, but neither view accounts for the entirety of the results observed here. Both suggest that past learning can influence new learning, but neither provides a specific mechanism by which past processes or assumptions can be changed. Ultimately, it will be important for theories of learning to posit specific mechanisms that control how and when pretraining will affect future processing of novel stimuli. The results presented here suggest that any specific mechanisms that are developed to account for the effect of subadditive pretraining should provide rules for generalization that incorporate temporal CS characteristics (Experiment 3), context specificity (Experiment 1), and retention interval (Experiment 2).

Within the framework of the inferential view, it might be simplest to posit that an acquired assumption of subadditivity is limited in the same way that an acquired association is limited. There might be some generalization of prior learning to similar stimuli, but that generalization is affected by the many factors that can affect similarity. This notion explains the results of Experiment 3, but not necessarily those of Experiments 1 and 2. As is noted in the Results and Discussion section of Experiment 2, the generalization of subadditive pretraining need not be directly related to the generalization of simple conditioned responding. In fact, there may be situations in which subadditive pretraining becomes less effective, whereas conditioned responding generalizes more. Specifically, an acquired stimulus attribute such as subadditivity might generalize less or be more readily forgotten than a CS-US association. This potentially explains why a context shift reduced the effect of subadditive pretraining on blocking, but similar shifts traditionally have little effect on simple conditioned fear (Bouton \& King, 1983; Hall \& Honey, 1989). It would also explain why a retention interval hindered the generalization of subadditive pretraining in our experiments and enhanced the generalization of conditioned responding in other studies (Riccio et al., 1992). A similar but slightly different view of the fragility of subadditivity training is suggested by the observation that unambiguous associative learning generalizes widely across test contexts and retention intervals, whereas extinction learning does not generalize widely from the place (i.e., context) and time of extinction. Extinction is sometimes viewed as learning an exception to the rule established during acquisition. So the context specificity of extinction may be regarded as arising from the fact that extinction involves an exception to a rule, the rule itself being less sensitive to context (because rules are, by definition, more general than exceptions). Now, given that subadditive pre- training might also serve as an exception to the default rule of additivity of cues (see Beckers et al., 2006), it is not surprising that such an exception to the putative rule of additivity would also be largely localized to the context in which subadditivity was encountered.

Finally, it is important to address the larger implications of the present experiments. We began this series of studies with limited expectations concerning the efficacy of any of our manipulations, but each manipulation that was employed attenuated the effectiveness of subadditive pretraining to some extent. One potential conclusion that might be drawn from the present experiments is that the pretraining effect observed here is so fragile that it is relatively unimportant to the field of learning. However, we do not subscribe to this idea. The basic blocking effect observed in Experiment 1 was very robust, and none of our manipulations produced a comparable deficit in responding. Thus, there is no indication that there was a complete elimination of the effect of subadditive pretraining in Experiments 1, 2, and 3. Furthermore, we contend that the present results are a reflection of the importance of the ability to flexibly express learning. To a large extent, the adaptive benefit of learning is determined by the appropriate balance of generalization and discrimination. In order for learning to be pragmatic, acquired information should generalize to similar situations, but novel situations should be approached with relatively fewer acquired presumptions. By applying past learning about stimulus interactions to slightly different situations, animals can save the time, cognitive effort, and potential risk involved in acquiring new information. Overgeneralizing presents its own risks, such as stifling new learning. The ability to form and appropriately use analogy is a key facet of human intelligence (Gentner, 2003), and may play no small role in nonhuman animal intelligence.

\section{AUTHOR NOTE}

NIMH Grant 33881 provided support for this research. Tom Beckers is a postdoctoral fellow of the Research Foundation-Flanders (FWO) We thank Jeffrey C. Amundson, Jonah Grossman, David Guez, Rachael Hessner, Olga Lipatova, Alyssa Orinstein, Gonzalo Urcelay, Kouji Urushihara, and James Witnauer for comments on an earlier version of the article. In addition, we are indebted to Jim Esposito for his aid in collecting the data. Correspondence concerning this article should be addressed to R. R. Miller, Department of Psychology, Binghamton University, Binghamton, NY 13902-6000 (e-mail: rmiller@ binghamton.edu).

\section{REFERENCES}

Alvarado, M. C., \& Rudy, J. W. (1992). Some properties of configural learning: An investigation of the transverse-patterning problem. Journal of Experimental Psychology: Animal Behavior Processes, 18, 145-153.

Beckers, T., De Houwer, J., Pineño, O., \& Miller, R. R. (2005). Outcome additivity and outcome maximality influence cue competition in human causal learning. Journal of Experimental Psychology: Learning, Memory, \& Cognition, 31, 238-249.

Beckers, T., Miller, R. R., De Houwer, J., \& Urushihara, K. (2006). Reasoning rats: Forward blocking in Pavlovian animal conditioning is sensitive to constraints of causal inference. Journal of Experimental Psychology: General, 135, 92-102.

Bouton, M. E. (1993). Context, time, and memory retrieval in the interference paradigms of Pavlovian learning. Psychological Bulletin, 114, 80-99. 
Bouton, M. E., \& King, D. A. (1983). Contextual control of the extinction of conditioned fear: Tests for the associative value of the context. Journal of Experimental Psychology: Animal Behavior Processes, 9, 248-265.

De Houwer, J., \& BeCKers, T. (2003). Secondary task difficulty modulates forward blocking in human contingency learning. Quarterly Journal of Experimental Psychology, 56B, 345-357.

De Houwer, J., Beckers, T., \& Glautier, S. (2002). Outcome and cue properties modulate blocking. Quarterly Journal of Experimental Psychology, 55A, 965-985.

De Houwer, J., Vandorpe, S., \& Beckers, T. (2005). On the role of controlled cognitive processes in human associative learning. In A. J. Wills (Ed.), New directions in human associative learning (pp. 4163). Mahwah, NJ: Erlbaum.

EscobAR, M., \& Miller, R. R. (2003). Timing in retroactive interference. Learning \& Behavior, 31, 257-272.

Estes, W. K. (1950). Toward a statistical theory of learning. Psychological Review, 57, 94-107.

Gentner, D. (2003). Why we're so smart. In D. Gentner \& S. GoldinMeadow (Eds.), Language in mind: Advances in the study of language and thought (pp. 195-235). Cambridge, MA: MIT Press

GodDEN, D. R., \& BADDELEY, A. D. (1975). Context-dependent memory in two natural environments: On land and underwater. British Journal of Psychology, 66, 325-331.

Hall, G., \& Honey, R. C. (1989). Contextual effects in conditioning, latent inhibition, and habituation: Associative and retrieval functions of contextual cues. Journal of Experimental Psychology: Animal Behavior Processes, 15, 232-241.

Hall, G., \& Honey, R. C. (1990). Context-specific conditioning in the conditioned-emotional-response procedure. Journal of Experimental Psychology: Animal Behavior Processes, 16, 271-278.

Hinchy, J., Lovibond, P. F., \& TeR-Horst, K. M. (1995). Blocking in human electrodermal conditioning. Quarterly Journal of Experimental Psychology, 48B, 2-12.

Holland, P. C. (1992). Occasion setting in Pavlovian conditioning. In D. L. Medin (Ed.), The psychology of learning and motivation (Vol. 28, pp. 69-125). San Diego: Academic Press.

Kamin, L. J. (1968). "Attention-like" processes in classical conditioning. In M. R. Jones (Ed.), Miami Symposium on the Prediction of Behavior: Aversive stimulation (pp. 9-31). Miami, FL: University of Miami Press.

Lachnit, H., Kimmel, H., Bevill, M., Martin, I., Levey, A., \& Haмm, A. (1990). Classical conditioning with human subjects. In P. J. D. Drenth, J. A. Sergeant, \& R. J. Takens (Eds.), European perspectives in psychology: Vol. 1. Theoretical, psychometrics, personality, developmental, educational, cognitive, gerontological (pp. 353368). Chichester, U.K.: Wiley.

Livesey, E. J., \& BoAKes, R. A. (2004). Outcome additivity, elemental processing and blocking in human causality judgements. Quarterly Journal of Experimental Psychology, 57B, 361-379.

Lovibond, P. F., Preston, G. C., \& Mackintosh, N. J. (1984). Context specificity of conditioning, extinction, and latent inhibition. Journal of Experimental Psychology: Animal Behavior Processes, 10, 360-375.

Melchers, K. G., ShanKs, D. R., \& Lachnit, H. (2008). Stimulus coding in human associative learning: Flexible representations of parts and wholes. Behavioural Processes, 77, 413-427.
Melchers, K. G., ÜNGÖR, M., \& LACHNIT, H. (2005). The experimental task influences cue competition in human causal learning. Journal of Experimental Psychology: Animal Behavior Processes, 31, 477-483.

MilLeR, R. R., \& Matzel, L. D. (1988). The comparator hypothesis: A response rule for the expression of associations. In G. H. Bower (Ed.), The psychology of learning and motivation (Vol. 22, pp. 51-92). New York: Academic Press.

Mitchell, C. J., \& Lovibond, P. F. (2002). Backward and forward blocking in human electrodermal conditioning: Blocking requires an assumption of outcome additivity. Quarterly Journal of Experimental Psychology, 55B, 311-329.

Pearce, J. M. (1994). Similarity and discrimination: A selective review and a connectionist model. Psychological Review, 101, 587-607.

Pearce, J. M., \& Hall, G. (1980). A model for Pavlovian learning: Variations in the effectiveness of conditioned but not of unconditioned stimuli. Psychological Review, 87, 532-552.

Pellón, R., \& Montaño, J. M. G. (1990). Conditioned stimuli as determinants of blocking in human electrodermal conditioning. In P. J. D. Drenth, J. A. Sergeant, \& R. J. Takens (Eds.), European perspectives in psychology: Vol. 2. Clinical, health, stress and anxiety, neuropsychology, psychophysiology (pp. 409-423). Chichester, U.K.: Wiley.

Rescorla, R. A., \& Wagner, A. R. (1972). A theory of Pavlovian conditioning: Variations in the effectiveness of reinforcement and nonreinforcement. In A. H. Black \& W. F. Prokasy (Eds.), Classical conditioning II: Current research and theory (pp. 64-99). New York: Appleton-Century-Crofts.

Riccio, D. C., Ackil, J., \& Burch-Vernon, A. (1992). Forgetting of stimulus attributes: Methodological implications for assessing associative phenomena. Psychological Bulletin, 112, 433-445.

Riccio, D. C., Richardson, R., \& EbNer, D. L. (1984). Memory retrieval deficits based upon altered contextual cues: A paradox. Psychological Bulletin, 96, 152-165.

ShanKs, D. R., \& Dickinson, A. (1987). Associative accounts of causality judgment. In G. H. Bower (Ed.), The psychology of learning and motivation (Vol. 21, pp. 229-261). New York: Academic Press.

Williams, D. A., \& BRAKER, D. S. (1999). Influence of past experience on the coding of compound stimuli. Journal of Experimental Psychology: Animal Behavior Processes, 25, 461-474.

Williams, D. A., \& BraKeR, D. S. (2002). Input coding in animal and human associative learning. Behavioural Processes, 57, 149-161.

Williams, D. A., Sagness, K. E., \& McPhee, J. E. (1994). Configural and elemental strategies in predictive learning. Journal of Experimental Psychology: Learning, Memory, \& Cognition, 20, 694-709.

\section{NOTE}

1. Forgetting can be caused by many theoretical mechanisms (e.g., trace decay, disrupted consolidation, interference, etc.). We do not intend to show favor toward any specific mechanism in the use of forgetting here.

(Manuscript received May 6, 2008; accepted for publication May 15,2008 .) 